ARTICLE

\title{
Genome-wide association study identifies 143 loci associated with 25 hydroxyvitamin D concentration
}

Joana A. Revez (10) 1, Tian Lin (10) 1, Zhen Qiao', Angli Xue (1) 1, Yan Holtz², Zhihong Zhu', Jian Zeng (1) 1, Huanwei Wang (10 1, Julia Sidorenko (1) 1, Kathryn E. Kemper', Anna A. E. Vinkhuyzen', Julanne Frater ${ }^{2}$, Darryl Eyles 2,3, Thomas H. J. Burne (1) 2,3, Brittany Mitchell (10) 4,5, Nicholas G. Martin (iD ${ }^{4}$, Gu Zhu4, Peter M. Visscher (10 1, Jian Yang (1) ${ }^{1,6}$, Naomi R. Wray (1) ${ }^{1,2,8 凶}$ \& John J. McGrath (1) 2,3,7,8凶

Vitamin D deficiency is a candidate risk factor for a range of adverse health outcomes. In a genome-wide association study of 25 hydroxyvitamin D (25OHD) concentration in 417,580 Europeans we identify 143 independent loci in 112 1-Mb regions, providing insights into the physiology of vitamin D and implicating genes involved in lipid and lipoprotein metabolism, dermal tissue properties, and the sulphonation and glucuronidation of 250HD. Mendelian randomization models find no robust evidence that $250 \mathrm{HD}$ concentration has causal effects on candidate phenotypes (e.g. BMI, psychiatric disorders), but many phenotypes have (direct or indirect) causal effects on $250 \mathrm{HD}$ concentration, clarifying the epidemiological relationship between $250 \mathrm{HD}$ status and the health outcomes examined in this study.

\footnotetext{
${ }^{1}$ Institute for Molecular Bioscience, The University of Queensland, Brisbane, QLD, Australia. ${ }^{2}$ Queensland Brain Institute, The University of Queensland, Brisbane, QLD, Australia. ${ }^{3}$ Queensland Centre for Mental Health Research, The Park Centre for Mental Health, Wacol, QLD, Australia. ${ }^{4}$ QIMR Berghofer Medical Research Institute, Brisbane, QLD, Australia. ${ }^{5}$ School of Biomedical Sciences, Faculty of Health, and Institute of Health and Biomedical Innovation, Queensland University of Technology, Brisbane, QLD, Australia. ${ }^{6}$ Institute for Advanced Research, Wenzhou Medical University, Wenzhou, Zhejiang 325027, China. ${ }^{7}$ National Centre for Register-based Research, Aarhus University, Aarhus, Denmark. ${ }^{8}$ These authors contributed equally: Naomi R. Wray, John J. McGrath.凶email: naomi.wray@uq.edu.au; j.mcgrath@uq.edu.au
} 
$\mathrm{n}$ recent decades, there has been considerable interest in the links between vitamin $\mathrm{D}$ concentration and general health. While classically linked to bone disorders, there is growing evidence to suggest that suboptimal vitamin $\mathrm{D}$ status may be a risk factor for a much wider range of adverse health outcomes ${ }^{1}$. Vitamin $\mathrm{D}$, the sunshine hormone, is the precursor of a secosteroid transcription regulator that operates via a nuclear receptor, and like other steroid hormones, exerts transcriptional control over many regions of the genome across many different tissues. In environments with access to adequate sunshine, ultraviolet radiation on the skin converts a precursor of cholesterol to vitamin $\mathrm{D}_{3}$. This is then further converted to 25hydroxyvitamin $\mathrm{D}_{3}$ (25OHD; used in assays of general vitamin $\mathrm{D}$ status), and then to the active hormone 1,25-dihydroxyvitamin $\mathrm{D}_{3}(1,25 \mathrm{OHD})$ in a variety of tissues. Some foods and vitamin $\mathrm{D}$ supplements also contribute to vitamin D levels. Definitions of vitamin $\mathrm{D}$ deficiency (e.g., $<25 \mathrm{nmol} \mathrm{L}^{-1}$ of $25 \mathrm{OHD}$ ) are predominantly based on bone health ${ }^{2}$-according to these definitions, vitamin $\mathrm{D}$ deficiency is common in many countries, regardless of latitude and economic status ${ }^{3}$.

Environmental factors such as season of testing and latitude contribute substantially to the serum concentration of $25 \mathrm{OHD}$ (lower in winter/spring; lower at higher latitudes) ${ }^{1}$. With respect to the genetic architecture of $25 \mathrm{OHD}$, twin and family studies have reported a wide range of heritability estimates (from $0 \%{ }^{4}$ to $90 \% 5$ ). A recent multivariate twin study demonstrated that approximately half of the total additive genetic variation in $25 \mathrm{OHD}$ may reflect genetic variation in skin colour and sun exposure behaviour ${ }^{6}$. Genome-wide association studies (GWAS) have identified common single-nucleotide polymorphisms (SNPs) located in biologically plausible genes ${ }^{7}$. The largest GWAS to date $(N=79,366)$ reported six significant loci, which include $G C$ (the vitamin D-binding protein gene), the DHCR7/NADSYN1 region (DHCR7 is involved in a conversion of a $25 \mathrm{OHD}$ precursor molecule to cholesterol) and CYP2R1 and CYP24A1 genes (which encode enzymes involved in 25OHD metabolism ${ }^{8}$ ). In total, common SNPs explain 7.5\% (standard error (s.e.) 1.9\%) of the variance of $25 \mathrm{OHD}^{8}$.

Here, we conduct a GWAS of $25 \mathrm{OHD}$ based on the large UK Biobank (UKB) sample ${ }^{9}$ and conduct a suite of post-GWAS analyses to aid interpretation of the results (Fig. 1). We present models that explore the genetic or causal relationship between body mass index (BMI) and 25OHD (high BMI is associated with lower $25 \mathrm{OHD}$ concentration in observational studies ${ }^{10}$ ). Because we have an interest in the association between 25OHD and psychiatric disorders ${ }^{11}$, we use Mendelian randomisation methods to investigate the bidirectional association between 25OHD and psychiatric disorders, as well as with a wider range of traits and diseases. In addition, we present a GWAS to identify loci associated with variance in $25 \mathrm{OHD}$ (i.e., variance quantitative trait locus (vQTL) analysis) which can identify putative genotype environment interactions without prior identification of the environmental effect ${ }^{12}$. We identify 143 independent loci in 112 $1-\mathrm{Mb}$ regions associated with $25 \mathrm{OHD}$ concentration, and our findings implicate genes involved in lipid metabolism, dermal tissue properties and conjugation of $25 \mathrm{OHD}$. We find no robust evidence that $25 \mathrm{OHD}$ concentration has causal effects on candidate phenotypes. However, we show that many phenotypes have (direct or indirect) causal effects on 25OHD concentration.

\section{Results}

25OHD phenotype. In total, 417,580 European UKB participants had both measures of vitamin D 25OHD and genome-wide genotypes ("Methods"). The distribution of 25OHD concentration, in keeping with expectation, is right skewed (Supplementary Fig. 1a), and showed the expected seasonal fluctuation (Supplementary Fig. 1b-d), with median, mean and interquartile range of $47.9,49.6,33.5-63.2 \mathrm{nmol} \mathrm{L}^{-1}$ (Supplementary Table 1). Covariates of age, BMI, genotyping batch, assessment centre, month of testing, supplement intake and the first four ancestry principal components (PCs), but not sex, were all significantly associated with 25OHD (Supplementary Table 1). Month of testing accounts for $14 \%$ of the variance of $25 \mathrm{OHD}$. Subsequent analyses use 25OHD after rank-based inverse-normal transformation (RINT), unless otherwise stated.

Heritability and SNP-based heritability. Our UKB sample included a set of 58,738 individuals related with coefficient of relationship $(r)>0.2$ to at least one other person in the set (all relatives), from whom we estimate the heritability of $25 \mathrm{OHD}$ to be 0.32 (s.e. $=0.01$ ) with little evidence for inflation from shared family environment (Fig. 2; Supplementary Fig. 2, Supplementary Data 1, Supplementary Note 1). The SNP-based heritability estimate $\left(\hat{h}_{\mathrm{SNP}}^{2}\right)$, which captures the genetic contribution from common (minor allele frequency or MAF > 0.01) variants, was 0.13 (s.e. $=0.01$ ) (see Supplementary Fig. 2, Supplementary Data 1 for a comparison of $\hat{h}_{\mathrm{SNP}}^{2}$ estimated from various methods). $\hat{h}_{\mathrm{SNP}}^{2}$ was significantly higher $\left(P=1.5 \times 10^{-3}\right.$; $Z$-test difference between two estimates, H0:difference $=0$ ) when estimated only from individuals measured for 25OHD in summer months (June to October) compared with those measured in winter months (December to April) $(0.19$, s.e. $=0.02$ vs. 0.10 , s.e. $=0.02$ ) (Fig. 2), as found for estimates of twin heritability ${ }^{6}$. The genetic correlation between the seasons was 0.80 (s.e. $=0.11$ ), not significantly different from 1 . The proportion of SNPs estimated to have an effect on the trait (polygenicity parameter) using the SBayesS method ${ }^{13}$ was $0.8 \%$ or 9000 SNPs of the $\sim 1.1$ million HapMap3 panel $^{14}$ common SNPs (Supplementary Table 2), much lower than estimates for most complex traits ${ }^{13}$. The SBayesS $S$ parameter, which describes the effect size-MAF relationship, was estimated as -0.78 (s.e. $=0.04$; Supplementary Table 3 ), consistent with a model of negative selection on the genetic variants associated with $25 \mathrm{OHD}$ levels (the magnitude of $S$ is higher than those of most complex traits studied ${ }^{13}$ ). Estimation of $\hat{h}_{\mathrm{SNP}}^{2}$ partitioned into ten components based on five MAF bins (each median split by linkage disequilibrium score) did not provide strong evidence for an increased role for less common variants, given the s.e. of estimates (Supplementary Fig. 3). Despite a strong phenotypic association between $25 \mathrm{OHD}$ and BMI of $-0.76 \mathrm{nmol}$ $\mathrm{L}^{-1}$ per BMI unit $(-0.036 \mathrm{RINT}(25 \mathrm{OHD})$ standard deviation (SD) units per BMI unit, linear regression $\left.P<2.2 \times 10^{-16}\right)$ and a phenotypic correlation of -0.17 (Supplementary Table 1), the estimates of heritability (both family and SNP-based) were hardly impacted when BMI was included as a covariate (Supplementary Fig. 2).

Genome-wide association study (GWAS) analysis. Given the potential for collider bias from using a heritable trait as a covariate $^{15}$, we conducted GWAS for $25 \mathrm{OHD}$ with and without BMI as a covariate. We also used $\mathrm{mtCOJO}^{16}$ to estimate the $25 \mathrm{OHD}$ SNP effects conditioning on those estimated for BMI from UKB data $^{17}$, a summary-data-based conditional analysis approach that was shown in simulations to be robust to collider bias when conditioning on a correlated trait ${ }^{16}$. Results were comparable across the three levels of BMI adjustment (Supplementary Data 2), so we report those with no correction for BMI, using results from all three analyses when this aids interpretation of the 


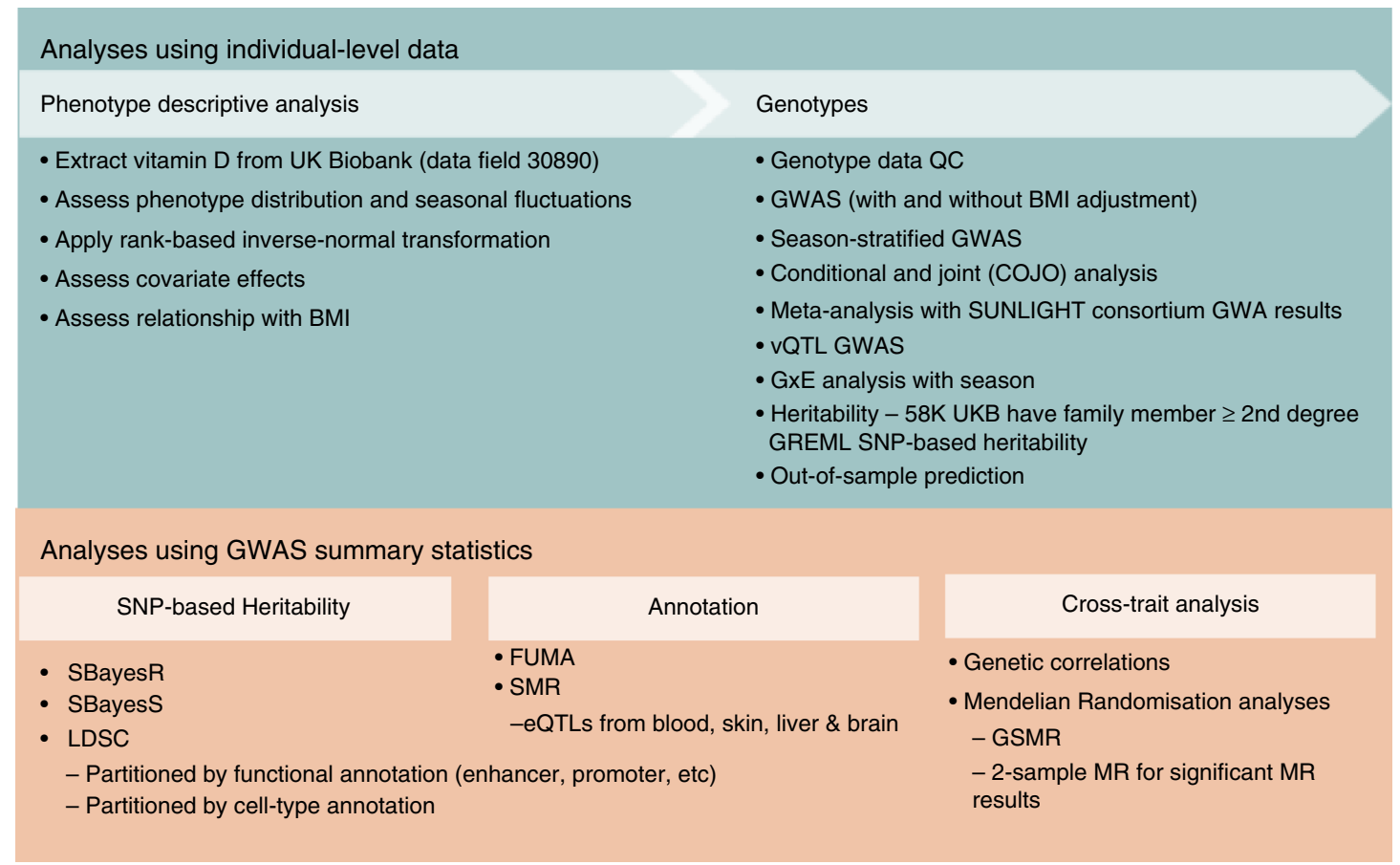

Fig. 1 Outline of key analytic steps described in this study. BMI body mass index, eQTL expression quantitative trait locus, FUMA functional mapping and annotation ${ }^{23}$, GREML ${ }^{69}$ genomic relationship restricted maximum likelihood68, GSMR generalised summary-based MR, GWAS genome-wide association study, GxE genotype-by-environment interaction, LDSC linkage disequilibrium score regression, MR Mendelian Randomisation, SMR summary-based $M R^{26}, Q C$ quality control, UKB UK Biobank, vQTL variance quantitative trait locus ${ }^{12}$.

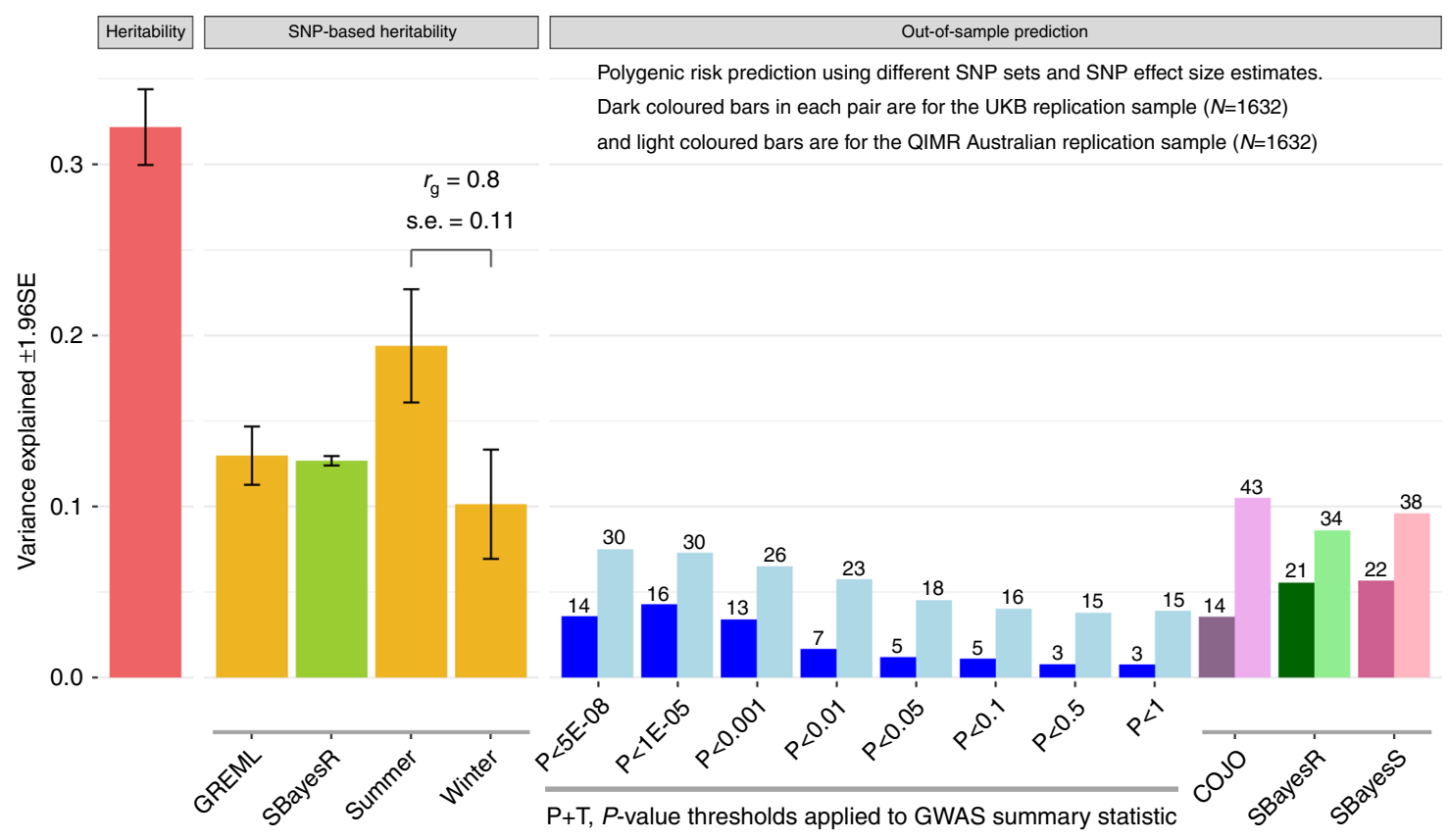

Fig. 2 Heritability, SNP-based heritability and variance explained in out-of-sample prediction. Heritability (left panel) and SNP-based heritability (middle panel) estimates and the variance explained in out-of-sample prediction (right panel). Heritability and SNP-based heritability estimates are presented with 95\% confidence interval. GCTA-GREML was used to estimate heritability from a UKB subset that included all pairs of individuals related with coefficient of relationship > 0.2 ( $N=58,738$ relatives). GCTA-GREML was used to estimate SNP-based heritabilities labelled GREML summer or winter using samples of $\sim 50 \mathrm{~K}$ participants randomly drawn from the UKB. The SBayesR SNP-based heritability is estimated from the GWAS summary statistics (N=417,580). In out-of-sample prediction into the QIMR and the UKB replication (UKBR) samples, polygenic risk scores (PRS) calculated by the standard P-value threshold method $(P+T)$ were outperformed by using SNP effect estimates calculated from GWAS summary statistics using the SBayesR or SBayesS methods. Bars of the same colour used the same methodology (noting that SBayesR generates an estimate of SNP-based heritability as well as SNP effect sizes in prediction analysis). The numbers on top of the bars are - log10 P-value of the regression of $250 H D$ on $250 H D$ PRS. COJO conditional and joint, $r g$ genetic correlation, s.e. standard error. 


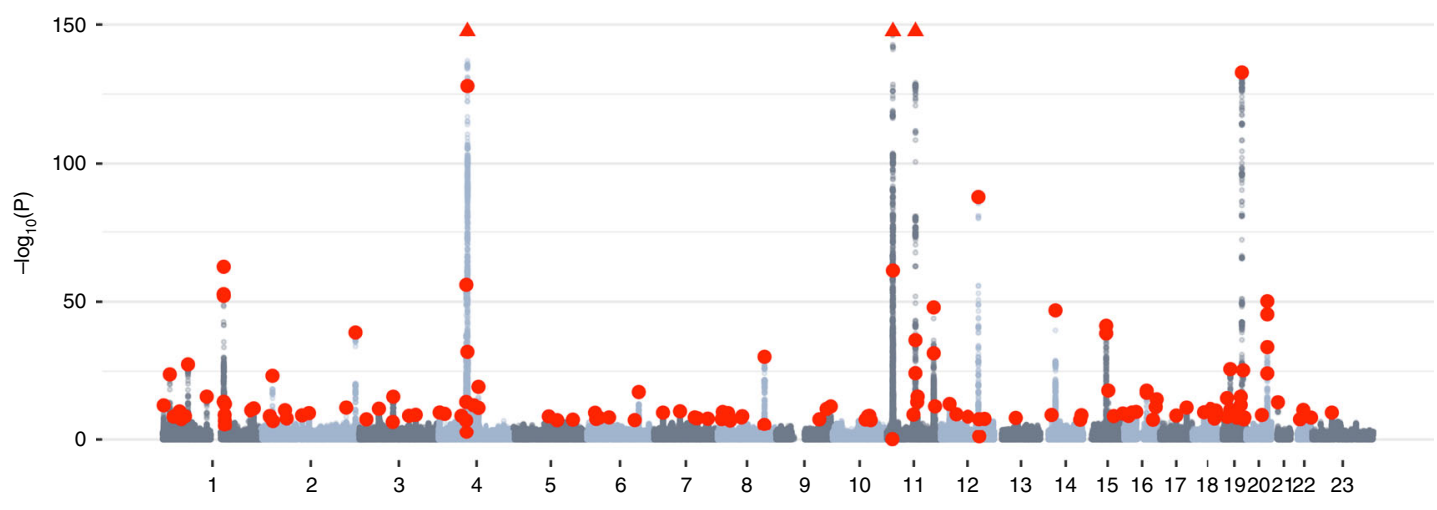

Fig. 3 Manhattan plot of the 250HD GWAS in the UK Biobank. Manhattan plot showing the $-\log 10$ P-values from the fastGWA62 association test of 25-hydroxyvitamin D (25OHD) with genome-wide SNPs. Red dots represent independent variants identified as genome-wide significant with conditional and joint analysis $\left(\mathrm{COJO}^{18}\right)$ applied to the GWAS summary statistics. The horizontal axis shows each chromosome, with 23 representing the $X$ chromosome. The vertical axis is restricted to $-\log 10 P$-values $<150$. Five COJO SNPs were associated at $P<1 \times 10^{-150}$ (four on chromosome 11 and one on chromosome 4; Supplementary Data 3) and have approximate locations represented by three red triangles at the top edge of the plot.

results. While there is some debate about the clinical threshold for vitamin $\mathrm{D}$ deficiency $\left(25 \mathrm{nmol} \mathrm{L}^{-1}\right.$ or $30 \mathrm{nmol} \mathrm{L}^{-1}$ serum concentration $)^{1,2}$, we chose the more conservative threshold of 25 $\mathrm{nmol} \mathrm{L}-1$. We conducted analyses bisecting 25OHD into a binary trait (less than $25 \mathrm{nmol} \mathrm{L}^{-1}, 25 \mathrm{nmol} \mathrm{L}^{-1}$ or greater), but the results were consistent (given the expected reduced power) with our reported results treating 25OHD as a quantitative trait (Supplementary Note 2).

A total of $8,806,780$ SNPs with MAF $>0.01$ were tested in the GWAS analysis. Of these, 18,864 were genome-wide significant (GWS; $P<5 \times 10^{-8}$ ). To identify independently associated loci, we applied the GCTA-COJO method ${ }^{18}$ to the GWAS summary statistics using LD between SNPs estimated from a UKB subset ("Methods"), and identified 143 independent loci (including one on chromosome X) (Fig. 3; Supplementary Data 3) in $1121-\mathrm{Mb}$ regions. Of these, 15 loci were low-frequency variants $(\mathrm{MAF}<$ 0.05 ), and 106 regions had no previously identified associations. All six loci reported in previous vitamin D GWAS $8,19,20$ were replicated in our study. While recognising that the COJO method cannot distinguish between SNPs in perfect LD, we note that within the 143 COJO independent variants: (a) 14 were nonsynonymous variants that alter protein coding (NRIP1, DSG1, TM6SF2, PLA2G3, GCKR, APOE, PCSK9, SEC23A, FLG, NPHS1, SDR42E1, CPS1, ADH1B, UGT1A5), and (b) 9 were annotated to include small insertion/deletions. A summary of the results is provided in Fig. 4, but are discussed later.

Summary statistics from the SUNLIGHT consortium ${ }^{8}$ were available for 2,579,297 SNPs, and the genetic correlation estimate with UKB results was not significantly different from $1\left(\hat{r}_{g}=0.92\right.$, s.e. $=0.06)$. Meta-analysis with our UKB GWAS results after imputation $^{21}$ of the SUNLIGHT summary statistics (Supplementary Methods) (6,912,294 overlapping SNPs) identified 15,154 GWS variants, 150 GCTA-COJO independent SNPs (Supplementary Methods, Supplementary Data 2). Of these, 91 were within $1-\mathrm{Mb}$ regions also identified in the UKB alone as GWS. Given that the meta-analysis only increased the total number of significant loci by seven, and given our preference not to include $\mathrm{BMI}$ as a covariate, we continued with the UKB-only results for our downstream analyses. See Supplementary Note 3 for further details.

Replication and out-of-sample genetic risk prediction. Of the 143 genome-wide significant COJO SNPs, 135, 135 and 108 were found in the QIMR $(N=1632)$, UKBR $(N=1632)$ and
SUNLIGHT $(N=79,366)$ consortium replication samples, respectively (Supplementary Data 3; Supplementary Note 3). Of these, $92\left(68 \%, P=6.7 \times 10^{-6}\right), 97\left(72 \%, P=7.5 \times 10^{-8}\right)$ and 89 $\left(82 \%, P=5.3 \times 10^{-13}\right)$ had the same sign of association test statistic as in the UKB discovery sample ( $P$-values from the binomial test with null hypothesis of random sign). The pairwise correlations between allele effect size estimates in the different cohorts were all highly significant, ranging from 0.44 between QIMR and UKBR and 0.91 between UKB and SUNLIGHT. Polygenic score prediction into the QIMR sample using SNP effects estimated in the UKB and the standard $P$-value thresholding method explained a maximum of $7.5 \%$ of the variance in RINT (25OHD residuals after regression on covariates) (Fig. 2; Supplementary Table 4) (linear regression $P=3.7 \times 10^{-31}$, at $P$ value threshold of $\left.P<5 \times 10^{-8}\right)$. When the polygenic scores were derived from SNP weights from COJO or SBayesR applied to the GWAS summary statistics ${ }^{13,22}$, the prediction variance was higher $10.5 \%$ and $9.6 \%$, respectively (Fig. 2; Supplementary Table 4). In the UKBR sample, the $P$-value thresholding method explained a maximum of $4.3 \%$ of variance $\left(P=3.2 \times 10^{-17}\right.$, at $P$ value threshold of $P<1 \times 10^{-5}$ ), while scores using the COJO SNP weights explained $3.6 \%$ of the variance $\left(P=1.6 \times 10^{-14}\right)$ and those using SBayesR SNP weights explain $5.5 \%$ of the variance $\left(P=5.7 \times 10^{-22}\right.$, Fig. 2; Supplementary Table 4).

Functional mapping and annotation of GWAS. To annotate the 25OHD GWAS, we first used the FUMA online pipeline ${ }^{23}$. Gene-set analyses showed that the top four pathways were related to glucuronidation, ascorbate and aldarate metabolism and uronic acid metabolism (Supplementary Data 4, 5). Keratinisation was the top Gene Ontology (GO) biological processes identified. Based on 53 tissue types from GTEx v6 $6^{24}$, the top tissues for differentially expressed genes identified in the GWAS were liver, brain and skin (sun exposed, and non-sun exposed; Supplementary Data 6). Partitioned SNP-based heritability analysis ${ }^{25}$ using cell-type-specific annotations identified five cell types (hepatocytes, two types of liver cells, skin cells and blood cells) at the nominal significance level of 0.05 (Supplementary Data 7), but none remained significant after correction for multiple testing (stratified LD score regression $P\left(P_{\text {LDSC }}\right)<$ $\left.2.4 \times 10^{-4}\right)$. In partitioned SNP-based heritability analysis using SNP annotation to 53 functional categories ${ }^{25}, 11$ passed multiple testing significance threshold $\left(P_{\text {LDSC }}<9.4 \times 10^{-4}\right.$; Supplementary Data 8) with a mix of annotations including 


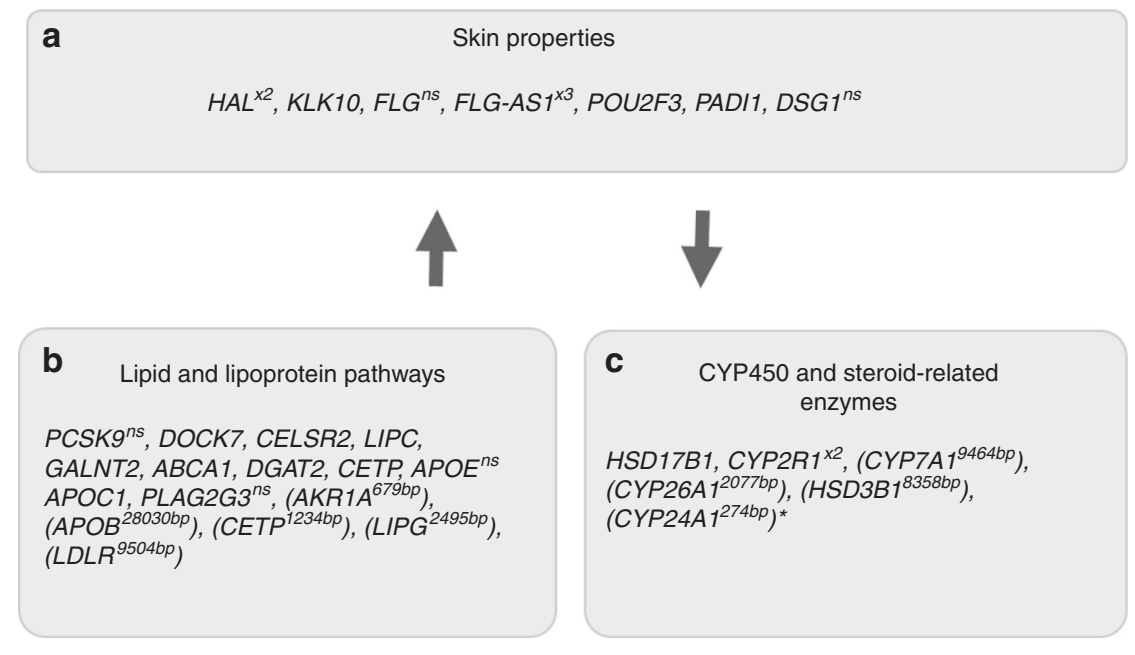

Selected loci of interest and putative mechanism related to Vitamin D pathways

- NRIP ${ }^{n s}$ : nuclear protein that interacts with nuclear receptors such as the vitamin D receptor

- SEC23A ${ }^{\text {ns: }}$ vesicle formation in endoplasmic reticulum

- EXOC4: exocyst formation required for docking on plasma membrane

- SDR42E $1^{\text {ns }}$ : short-chain dehydrogenase/reductase involved in the metabolism of steroids

- $A L D H 1 A 2^{x 2}:$ aldehyde dehydrogenase enzyme

- $A D H 1 A, A D H 1 B^{\text {ns }}$ : members of alcohol dehydrogenase family - metabolizes hydroxysteroids

- NPHS1 ${ }^{\text {ns }}$ : ultrafilter associated with exclusion of albumin and plasma macromolecules from urinary excretion. This may influence the excretion of the vitamin $\mathrm{D}$ binding protein, which will impact on the functional half-life of 25 hydroxyvitamin D

- $G C^{x 2}$ : vitamin $D$ binding protein - a highly polymorphic binding/transport protein that extends the functional half-life of 25 hydroxyvitamin $D$

- SULT21A: conjugates sulphur containing molecule to 25 hydroxyvitamin D (to produce 25 hydroxyvitamin D-3-sulfate) which can lead to excretion and/or recycling

- UGT1A4, UGT1A5 ${ }^{\text {ns }}$, UGT1A6, UGT1A7, UGT1A8, UGT1A9, UGT1A10, (UGT2B29P ${ }^{3077 b p}$ ): conjugates glucuronide molecule to 25 hydroxyvitamin $D$ which can lead to excretion and/or recycling

Fig. 4 Summary of selected variants associated with 250HD in the UK Biobank. Top panel shows loci associated with skin integrity, lipid and lipoprotein pathways and CYP450 and steroid-associated enzymes. Lower panel shows selected variants and putative mechanisms related to 25-hydroxyvitamin $\mathrm{D}(25 \mathrm{OHD})$ concentration. For selected inter-genic loci, the nearest (upstream or downstream) gene is shown in brackets. The distance between the loci and the nearest gene is shown in base pairs. ${ }^{*} \mathrm{CYP} 24 \mathrm{~A} 1$ was also the closest gene for an additional three inter-genic loci with distances between $32,865-55,282$ base pairs. ns non-synonymous variant, $x 2$ or $x 3$ two or three loci found within the gene. We note that nearest gene annotation should be interpreted recognising that these are not proof of a causal relationship between the associated SNP and expression of the gene (none-the-less supporting evidence for causal relationships is given by the SMR analyses for some loci).

transcription factor binding sites and transcription start sites (notable because vitamin $\mathrm{D}$ operates via a nuclear receptor, which binds to vitamin D response elements), as well as a role for repressed sites, conserved regions, enhancer and coding regions and histone modification marks.

To identify 25OHD SNP associations with statistical evidence consistent with a causal/pleiotropic association via gene expression, we used summary-data-based Mendelian randomisation $(\mathrm{SMR})^{26}$ using the 15,504 gene probes with significant cis-eQTLs identified from whole blood eQTLGen data ${ }^{27}$. After Bonferroni correction, we found 112 significantly associated gene expression probes $\left(P_{\mathrm{SMR}}<3.2 \times 10^{-6}\right.$, i.e., $0.05 / 15,504$, being the total number of probes tested in SMR analysis; Supplementary Data 9, Supplementary Fig. 4; full details of the SMR analyses can be found on https://cnsgenomics.com). These results are discussed in detail in Supplementary Note 4 , and add weight to the hypothesis that the SMR-identified eQTL variants may be causally related to $25 \mathrm{OHD}$ concentrations.
Putative causal relationships with other traits. First, we investigated the relationship between 25OHD and BMI. The LDSC ${ }^{28}$ genetic correlation estimated from 25OHD and BMI GWAS summary statistics was -0.17 (s.e. $=0.03$ ) (Supplementary Fig. 2, Supplementary Data 10). Bidirectional Mendelian randomisation $^{16}$ analysis provided strong support for the hypothesis that high $\mathrm{BMI}$ is causal for low $25 \mathrm{OHD}\left(b_{\mathrm{BMI} .25 \mathrm{OHD}}=-0.130\right.$; s.e. $=$ $0.005 ; P_{\mathrm{GSMR}}=4.7 \times 10^{-162}$; based on $1020 \mathrm{BMI}$-associated SNP instruments), with no support for a causal effect of vitamin $\mathrm{D}$ on BMI $\left(b_{25 \mathrm{OHD} . \mathrm{BMI}}=0.008\right.$; s.e. $=0.006 ; P_{\mathrm{GSMR}}=0.20$; based on 210 vitamin $\mathrm{D}$-associated SNPs) (these results were confirmed by other MR methods ${ }^{29}$; Supplementary Table 5). Notably, the HEIDI-outlier test in the GSMR analyses excluded 70 BMI and 67 25OHD SNP instruments, whose combination of SNP effect sizes likely reflects a pleiotropic relationship or confounding. Using the SNPs excluded by the HEIDI-outlier test, the estimates were $b_{\mathrm{BMI} .25 \mathrm{OHD}}=0.17 \quad\left(\right.$ s.e. $\left.=0.0182 ; \quad \mathrm{PGSMR}=1.2 \times 10^{-20}\right)$ and $b_{25 \mathrm{OHD} . \mathrm{BMI}}=-0.15$ (s.e. $\left.=0.017, P_{\mathrm{GSMR}}=2.7 \times 10^{-18}\right)$. Hence, 
despite the clear evidence for a causal relationship between high BMI and low 25OHD, the biological relationship between these traits is more complex.

Next, we estimated genetic correlations $\left(r_{g}\right)$ between 25OHD and 746 traits with GWAS summary statistics available in LD $\mathrm{Hub}^{30}$, and we used LDSC to estimate $r_{g}$ between 25OHD and 18 traits (including six psychiatric disorders) with GWAS summary statistics that are more recent than those included in LD Hub. Although many of the traits are highly correlated, we use a Bonferroni correction for 764 tests as the threshold for discussion of $r_{g}$. The LDSC regression intercepts were close to zero, suggesting no sample overlap (Supplementary Data 10), except for LD Hub traits derived from UKB analyses, where the intercept, as expected from theory, equates to the phenotypic correlation. We found significant associations between 25OHD and a range of brain-related phenotypes (including autism spectrum disorder, intelligence ${ }^{31}$, major depression, bipolar disorder and schizophrenia; Supplementary Fig. 5). Notably, the most significant $r_{g}$ were with cognitive-associated traits-for example, a negative correlation $\left(\hat{r}_{g}=-0.24\right.$, s.e. $=$ $0.03, \quad P_{\mathrm{H} 0: \mathrm{rg}=0}=1.6 \times 10^{-14}$ ) with intelligence. There was also a significant negative $r_{g}$ with hours spent using a computer $\left(\hat{r}_{g}=-0.22\right.$, s.e. $\left.=0.03, P_{\mathrm{H} 0: \mathrm{rg}=0}=5.1 \times 10^{-15}\right)$. These findings may be mediated by an association between higher intelligence and behaviour associated with less exposure to bright sunshine (and thus, lower 25OHD). Of note, behaviours associated with outdoor activity (duration of walks, duration of vigorous activity) were positively associated with $25 \mathrm{OHD}$, while phenotypes related to chronic disability were negatively associated with $25 \mathrm{OHD}$.

Next, we investigated if some of the significant genetic correlations could be explained by causal relationships using bidirectional GSMR models-here a more complex pattern of association emerged (Fig. 5; Supplementary Data 11). We found no evidence for putative causal effects between 25OHD and other traits; GSMR analyses without the HEIDI-outlier filtering step (Fig. 5a) suggest strong pleiotropy for some traits, such as dyslipidemia, coronary artery disease, intelligence and educational attainment. Finally, we examined the reciprocal relationship_if variants associated with a range of traits were directionally associated with $25 \mathrm{OHD}$. Regardless of the use of HEIDI filtering, and often regardless of adjustments for BMI, we found evidence consistent with increased risk of several traits or disorders being causal (directly or indirectly) with lower 25OHD concentrations (Fig. 5b). This was the case for intelligence, dyslipidemia, major depression, bipolar disorder, type 2 diabetes and schizophrenia. The findings might suggest these traits or disorders are associated with behaviours that lead to reduced production of 25OHD (e.g., less outdoor activity and physical activities). The GSMR findings were also checked with the portfolio of MR methods implemented in the two-sample MR (2SMR) software ${ }^{29}$ (Supplementary Data 12).

Gene-environment interplay. We conducted a genome-wide vQTL analysis, as implemented in OSCA $^{12}$ to identify SNPs associated with variance in 25OHD (not RINT-transformed). Such associations can reflect genotype-by-environment interaction in the absence of measurement, or indeed knowledge, of the interacting environmental risk factor ${ }^{12}$. Using data from 318,851 unrelated individuals of European ancestry, we tested 6,098,063 variants with MAF $>0.05$, and identified 4008 GWS vQTLs, of which 25 were independent (LD $r^{2}<0.01,5-\mathrm{MB}$ window), and several were in genes with previously described links to vitamin D-related pathways (e.g., GC, UGT2B7, SEC23A, SULT2A1, KLK10, NADSYN1). Of the 25 independent vQTLs, 23 were also QTLs (identified as genome-wide significant in the GWAS analysis) while the two non-QTL loci were still associated at $P_{\text {GWAS }}<10^{-5}$ (Supplementary Data 13 ). One was in the POR gene, which encodes a cytochrome p450 oxidoreductase that donates electrons from NADPH to cytochrome P450 enzymes (encoded by CYP450 genes), which are involved in vitamin D metabolism ${ }^{32}$. Variants in POR have previously been associated with coffee intake ${ }^{33}$. The other exclusive vQTL (rs1030431) is 12,126 bp upstream from $U B X N 2 B$; the SNP is significantly associated with gall bladder diseases and lipid metabolism traits in the $\mathrm{UKB}^{34}$.

An environmental factor with known association with $25 \mathrm{OHD}$ is the season of testing. To investigate if the associations between the vQTLs and the phenotypic variance of $250 \mathrm{HD}$ reflected gene-environment $(\mathrm{GxE})$ interactions with season of blood draw, we performed a GxE analysis with season (winter vs. summer). Of $6,098,063$ variants tested (MAF $>0.05), 1127$ had a GWS $(P<5 \times$ $\left.10^{-8}\right)$ interaction with season, and $1120(99 \%)$ were also GWS in the vQTL analysis. From the 1127 GWS interactions, five were independent (LD $r^{2}<0.01$, window $5 \mathrm{Mb}$ ) and were located in regions that have well-known vitamin D-related genes in chromosomes 11 (e.g., CYP2R1 region) and 14 (e.g., SEC23A) (Supplementary Data 14). Notably, of the 20 vQTL loci without significant GxE with season, at least half showed no evidence at all for GxE with season (Supplementary Fig. 6), so these variants are candidates for $\mathrm{GxE}$ with other environmental factors.

\section{Discussion}

We have identified 143 loci associated with 25OHD concentration. Recognising that only six associated loci had been reported to date, these discoveries provide important insights into previously unknown or poorly understood vitamin D-related pathways, and substantially increase our knowledge of the genetic correlates of $25 \mathrm{OHD}$ compared with previous studies ${ }^{7}$ (Fig. 4). First, the three most associated loci, all identified in previous studies $^{8}$, are noteworthy (chr4:rs1352846, chr11:rs116970203 and chr11:rs12794714, all with association test $P<1.0 \times 10^{-400}$, all with their minor allele reducing 25OHD). rs1352846 (MAF $=$ $0.29(\mathrm{G}))$ is in the $G C$ locus $^{8,20}$, which encodes a protein synthesised in the liver that binds to, and transports vitamin $\mathrm{D}$ and its metabolites. rs116970203 is a low-frequency variant $(\mathrm{MAF}=$ 0.03 (A)) located in intron 11 of the $P D E 3 B$ gene. It is also a perfect proxy for rs117913124 (LD $r^{2}=1$ ), a low-frequency synonymous coding variant in $C Y P 2 R 1$, which was previously reported to associate with $25 \mathrm{OHD}^{19}$. Another CYP2R1 synonymous variant was also identified (rs12794714; $\mathrm{MAF}=0.42(\mathrm{~A})$ ). CYP2R1 encodes a crucial hepatic enzyme involved in the hydroxylation of vitamin D to 25OHD. Given the complexity of the association pattern observed in chromosome 11, we confirmed the independence of the COJO identified variants using individual-level data (Supplementary Table 6). In line with previous findings ${ }^{19}$, the two-way conditional analysis showed that the effect of the low-frequency SNP (rs116970203 or rs117913124) and common SNP (rs12794714 or rs10741657) were largely independent.

Our findings provide convergent evidence that genes related to lipid- and lipoprotein-related pathways influence 25OHD concentration. In particular, we confirm a unidirectional relationship between SNP instruments that influence higher BMI and lower $25 \mathrm{OHD}$ concentration, but not the reciprocal relationship. This relationship exists against a background of a highly intercorrelated pattern of relationships between genes that influence both $25 \mathrm{OHD}$ and a wide range of lipid-related metabolic phenotypes. There were variants within genes with well-described functions related to lipid and lipoprotein-related pathways ${ }^{35}$ (e.g., PCSK9, DOCK7, CELSR2, GALNT2, ABCA1, DGAT2, CETP, 


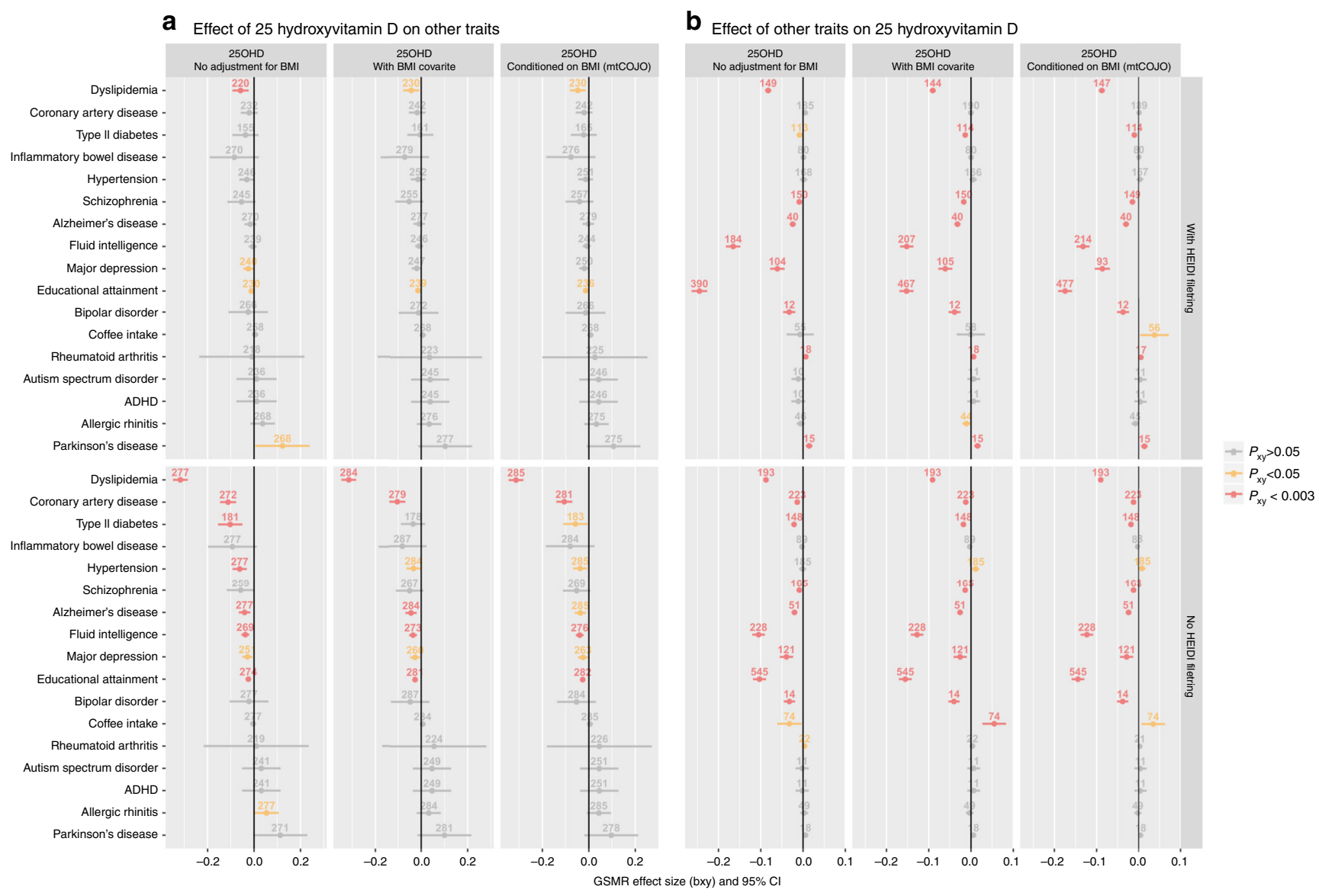

Fig. 5 Mendelian randomisation analysis estimates from GWAS results from 250HD and selected phenotypes. Bidirectional Generalised Summary-data level Mendelian Randomisation (GSMR) between 25-hydroxyvitamin D (25OHD) concentrations and selected phenotypes, by three types of adjustments for body mass index (BMI) and with/without HEIDI filtering of pleiotropic loci. Panel a shows the estimate of the causal effect estimate $\left(\hat{b}_{x y} ;\right.$ dots), and $95 \%$ confidence intervals (bars), of $250 \mathrm{HD}$ concentration on selected phenotypes. $\hat{b}_{x y}$ (and respective $P$-values) was obtained with the generalised summary-data-based Mendelian randomisation (GSMR) method ${ }^{16}$. Negative $\hat{b}_{x y}$ indicates that variants associated with increased $25 \mathrm{OHD}$ concentration were associated with a smaller value/reduced risk for the phenotypes of interest. Panel $\mathbf{b}$ shows the estimate of the causal effect (and $95 \%$ confidence intervals) of the same selected phenotypes on $250 \mathrm{HD}$ concentration. Results are presented with (upper half) and without (lower half) filtering of pleiotropic associations with the heterogeneity in dependent instruments (HEIDI) test, respectively. The numbers above each effect size indicate the number of SNP instruments used in each analysis. For each set of analyses, we show GWAS results: (i) without adjustment for body mass index (BMI), (ii) with $\mathrm{BMI}$ included as a covariate and (iii) conditioned on BMI using $\mathrm{mtCOJO}^{16}$. The GMSR estimates and $95 \%$ confidence intervals are shown in three colours according to the $P$-value thresholds: grey (non-significant; PGSMR $>0.05$ ), yellow (nominally significant; PGSMR $<0.05$ ) and red (significant after Bonferonni correction for multiple testing; PGSMR $<0.003$ ). All $\hat{b}_{x y}$, respective standard errors and $P$-values can be found in tabular form in Supplementary Data 11.

APOE, APOC1, PLA2G3). In addition, several inter-genic loci had closest upstream or downstream genes of interest to lipid and lipoprotein pathways (AKR1A, APOB, CETP, LIPG, LDLR). Variants in these genes influence overall lipid concentrations, including the concentration of 7-dehydrocholesterol in the skin. We identified a locus (chr11:rs12803256) in an uncharacterised RNA gene (FLJ42102) 11,057 base pairs upstream from DHCR7. This region has been identified in previous GWAS studies, and DHCR7 is a strong candidate gene because of its known role in the conversion of 7-dehydrocholesterol in the skin to pre-vitamin $\mathrm{D}_{3}$. We note that the broad region on Chr11 containing DHCR7 and NADSYN1 included several loci of interest according to both GCTA-COJO and SMR analyses-this complex area warrants additional research.

The GWAS uncovered a range of previously unreported findings, indicating that properties of the skin not related to pigmentation are associated with $25 \mathrm{OHD}$ concentration. While it is well known that individuals with darker skin tend to have lower
25OHD (related to the melanin content in the skin blocking $(\mathrm{UVB})^{1}$, our findings provide evidence that SNPs associated with genes that influence dermal development (e.g., $P A D I)^{36}$ and integrity (e.g., FLG; FLG-AS1, POU2F3, KLK10, DSG1) 37,38 are also associated with $25 \mathrm{OHD}$ status. It has been suggested that variants in the FLG gene may have evolved in order to optimise $25 \mathrm{OHD}$ production at high latitude $\mathrm{e}^{39,40}$. HAL (histidine ammonia-lyase) codes for an enzyme that deaminates L-histidine to trans-uronic acid. The top SNP in this region (rs10859995) is within an intron of this gene. The gene is expressed in the skin, and is upregulated during keratinocyte differentiation ${ }^{41}$. It has been demonstrated that trans-urocanic acid in the stratum corneum can absorb $\mathrm{UVB}^{42}$ and can reduce the production $25 \mathrm{OHD}^{43}$. The MAGMA gene-set analysis ${ }^{23}$ also showed that variants associated the uronic acid pathways were significantly overrepresented in our findings (Supplementary Data 5). The concentration of trans-uronic acid varies widely between individuals $^{43,44}$, but is not related to skin colour/pigmentation ${ }^{44}$. It is 
important to note that our sample was restricted to Europeans, and analyses included 40 ancestry PCs as covariates, four of which were strongly associated with 25OHD (Supplementary Table 1). If these PCs capture variants related to skin colour within Europeans, these variants are less likely to be identified in our analyses. FUMA analyses did not identify an overrepresentation of variants known to be related to skin colour in our GWAS.

Our study expands the range of enzymes implicated in the synthesis and breakdown of vitamin D-related molecules. These include genes from the hydroxysteroid 17-beta dehydrogenase family ( $H S D 17 B 1, H S D 3 B 1)$, a family of short-chain dehydrogenases/reductases, which are involved in steroidogenesis and steroid metabolism. CYP2R1 is a key regulator of 25OHD status, via hepatic conversion of vitamin $\mathrm{D}$ to $25 \mathrm{OHD}$-two loci were found within this gene. Other members of this large family of enzymes associated with $25 \mathrm{OHD}$ concentrations include CYP7A1, CYP26A1 and CYP24A1.

We identified many variants within genes related to the modification of lipophilic molecules (including seco-steroids, such as 25OHD and related species). Associated regions on chromosomes 2 and 4 include enzymes in the UDPglucuronosyltransferase family, which are critical in the glucuronidation pathways. The involvement of these genes in the degradation and potential conjugate recycling of $25 \mathrm{OHD}$ has recently been described ${ }^{45,46}$. We identified variants in the SULT21A gene, which encodes the enzyme responsible for the sulphonation of $25 \mathrm{OHD}^{46,47}$. Our findings provide support for the hypothesis that these mechanisms influence $25 \mathrm{OHD}$ concentration. We identified variants in the SLCO1B1 gene, which encodes a transmembrane receptor that mediates the sodiumindependent uptake of numerous endogenous compounds, including sulfated steroid molecules ${ }^{48}$. It is not known if this mechanism is involved in the uptake of the sulphated 25OHD. It has been proposed that vitamin D may undergo conjugate cycling (e.g., bidirectional conversion between 25OHD and 25OHDsulphate $)^{49}$. A proportion of the total $25 \mathrm{OHD}$ may exist in the sulphated form, which could act as circulating reservoir for later de-sulfation in peripheral tissues. In addition, conjugated versions of $25 \mathrm{OHD}$ with glucuronide ${ }^{45}$ and sulfate ${ }^{46}$ have both been detected in bile, which suggests enterohepatic mechanisms may provide another reservoir that buffers total 25OHD reserves. The findings also have implications for how to assay total 25OHD reserves. Current extraction and assay techniques used to quantitate $25 \mathrm{OHD}$ are not optimised for sulphonated or glucuronidated species of $25 \mathrm{OHD}$, thus total $25 \mathrm{OHD}$ status may not accurately reflect the contribution of these conjugated species. In addition, these mechanisms would contribute to the functional half-life of $25 \mathrm{OHD}$, and thus influence vitamin D status during periods of reduced exposure to bright sunshine (e.g., during winter). Finally, variants in a range of previously unreported enzymatic pathways were also associated with 25OHD concentration (e.g., short-chain dehydrogenase/reductase, aldehyde dehydrogenase, alcohol dehydrogenase).

The large sample size afforded by the UKB sample provides a thorough description of the genetic architecture of 25OHD. The SNP-based heritability (which captures the contribution to variation between people associated with common DNA variants) estimated in the UKB was 0.13 (95\% CI: 0.12-0.14), which means that all genotyped/imputed variants with MAF $>0.01$ explain about $41 \%$ of the heritability estimated from close relatives (i.e., 0.13/0.32, SNP-based heritability/heritability, Fig. 2). The 143 loci represent only $1121-\mathrm{Mb}$ regions, with six of the $1-\mathrm{Mb}$ regions harbouring four loci each. The final set of 143 loci was achieved by applying the COJO (conditional and joint) algorithm onto the GWAS summary statistics using the linkage disequilibrium structure to account for the correlation structure between SNPs. Two regions on chromosome 11 are particularly complex, Supplementary Data 3). Polygenic score predictor using SNP effect weights estimated in the UKB explained up to $10.5 \%$ and $5.7 \%$ of variance (after accounting for covariates) in independent samples QIMR and UKB. Aside from sampling differences, the higher variance explained in the Australian QIMR sample is in line with the higher heritability estimated from family data (QIMR 0.50 (95\% CI: $0.38-0.64)$ vs 0.32 (95\% CI: $0.30-0.34)$ estimated in the UKB. One explanation for the difference is that the QIMR samples were predominantly recruited at latitude $27^{\circ} \mathrm{S}$; at this latitude, there is sufficient UVR to allow for vitamin D synthesis throughout the year.

We also identified 25 independent SNPs associated with variance in 25OHD - these are putative GxE loci. While five of these have strong evidence of interacting with season of measurement, at least ten are $\mathrm{GxE}$ candidates with yet-to-be-identified environmental risk factors, and search of published GWAS results for association with these SNPs (i.e., PheWAS ${ }^{34}$ ) may help with this prioritisation (Supplementary Data 13). In summer months, the mean 25OHD concentrations are higher, and a larger proportion of the variance could be attributed to genetic factors in summer compared with winter (SNP-based heritability of 0.19 , s.e. $=0.02$, vs 0.10 , s.e. $\left.=0.02, P_{\text {different }}=1.5 \times 10^{-3}\right)$, and this reflected an increase in genetic variance rather than a decrease in residual variance (Supplementary Data 1). However, the genetic correlation from summer and winter SNP effect sizes was not significantly different from 1 . Five loci were identified as significant in $\mathrm{GxE}$ analysis with season, and for two the direction of effect was reversed (Supplementary Data 14). The vitamin D phenotype is an interesting one to explore from the perspective of $\mathrm{GxE}$ as seasonal fluctuations provide a natural experiment to dissect components of the genetic architecture that influence synthesis (i.e., inflow) and excretion (i.e., outflow) of 25OHD-related pathways.

In the UKB participants, high BMI is associated with reduced $25 \mathrm{OHD}$ concentration, in keeping with a large body of observational epidemiology ${ }^{50}$. However, we did not find statistical evidence in support of a causal role for the 25OHD level on BMI. In contrast, there was evidence for pleiotropic effects of SNPs on the two traits as well as for high BMI being causal (directly or indirectly) for low 25OHD. Genetic correlations were significant between 25OHD concentration and a range of phenotypes (Fig. 5). However, in robust directional models, we found no evidence in support of a causal role for 25OHD concentration on these traits. Of interest, we found evidence that higher intelligence and an increased risk of several psychiatric disorders may cause reduced $25 \mathrm{OHD}$ concentrations. With respect to intelligence, this would be consistent with previous links between intelligence and years of education leading to working indoors, and subsequent lower concentrations of $25 \mathrm{OHD}^{51}$. One of our motivations for undertaking this study was to investigate the hypothesis of a causality relationship between 25OHD and psychiatric disorders $^{52}$. The Mendelian randomisation analyses conducted here do not support a causal role for $25 \mathrm{OHD}$ levels and these disorders, and hence the reported epidemiological associations could reflect confounding and/or reverse causation. Vitamin D deficiency is common in those with established psychiatric disorders, as a consequence of reduced outdoor behaviour ${ }^{53}$. It is feasible that the observed association between 25OHD concentration in blood spot samples taken at birth with later-life increased risk of schizophrenia ${ }^{11,54}$ could be confounded by outdoor behaviour of mothers, which may be correlated with the mother's genetic liability to schizophrenia. While we find no evidence to support the hypotheses that variants associated with low 25OHD concentrations were associated with any of the selected phenotypes, we note 
that there is a linearity assumption in our Mendelian randomisation analyses. In other words, if only very low concentrations of 25OHD are associated with adverse outcomes, then this nonlinear exposure-risk association may not be confidently detected (see Supplementary Note 2 for further discussion). As the genetic architecture of the broad range of vitamin D-related phenotypes becomes better understood, issues related to potential threshold effects (e.g., disease-specific thresholds for clinical deficiency) should be re-examined.

We have identified 143 loci associated with 25OHD concentration, and have provided new directions for vitamin D research. In particular, our findings suggest that pathways related to sulphonation and glucuronidation warrant closer scrutiny-for example, there may be a case to measure these modified species of 25OHD and related molecules in order to better understand vitamin D status. Our studies based on Mendelian randomisation do not support hypotheses that vitamin D concentration is associated with a broad range of candidate phenotypes, in particular, psychiatric disorders. The findings provide insights into the physiology of vitamin D and the relationship between $25 \mathrm{OHD}$ status and health.

\section{Methods}

The UK Biobank sample. The UK Biobank (UKB) is a large population cohort with phenotype, genotype and clinical information on more than 502,000 individuals (age range from 40 to 69 years old). Participants were registered with the National Health Service, and lived $\sim 25$ miles from one of the 22 recruitment centres across the UK ${ }^{9}$. Participants were recruited between 2006 and 2010 . Informed consent was obtained by UK Biobank from all participants, and the study was approved by the North West Multicentre Research Ethnics Service Committee. The participants of the study were not representative of the original sampling frame, with evidence of a healthy volunteer bias ${ }^{55}$

Genotype data were quality-controlled and imputed to the Haplotype Reference Consortium (HRC) $)^{56}$ and UK10K ${ }^{57}$ reference panels by the UKB group ${ }^{58}$. We extracted variants with minor allele count $(\mathrm{MAC})>5$ and imputation score $>0.3$ for all individuals, and converted genotype probabilities to hard-call genotypes using PLINK2 (--hard-call 0.1 $)^{59}$. Then, we excluded variants with genotype missingness $>0.05$, Hardy-Weinberg equilibrium test $P>1 \times 10^{-5}$, and minor allele frequency $(\mathrm{MAF})<0.01$. In total, 8,806,780 variants (hereafter SNPs, but could include small insertion/deletions (INDELS)), including 260,713 SNPs in the X chromosome, were available for analysis.

Individuals of European ancestry were identified by projecting the UKB sample to the first two principal components (PCs) of the 1000 Genome Project $\left(1 \mathrm{KGP}^{60}\right)$, using Hap Map 3 (HM3) SNPs with MAF $>0.01$ in both data sets. European ancestry was assigned based on $>0.9$ posterior probability of belonging to the $1 \mathrm{KGP}$ European reference cluster.

Assessment of 25-hydroxyvitamin D concentration. Vitamin D 25OHD levels were measured in blood samples collected at two instances: the initial assessment visit, conducted between 2006 and 2010, and a repeat assessment visit, conducted between 2012 and 2013. The Diasorin Liason ${ }^{\circledR}$, a chemiluminescent immunoassay (CLIA) was used for the quantitative determination of 25OHD. The assay measures total $25 \mathrm{OHD}$ concentration (i.e., $25 \mathrm{OHD}_{3}$ and $25 \mathrm{OHD}_{2}$ ). Participants with 25OHD concentrations below or above the validated range for the assay (10-375 $\mathrm{nmol} \mathrm{L} \mathrm{L}^{-1}$ ) were excluded. The average within-laboratory coefficient of variation (CV) (and standard deviation) ranged from $5.04(4.73)$ to $6.14(2.21)^{61}$

Of 502,536 UKB participants, 449,978 (90\%) had vitamin D 25OHD levels (data field 30890) measured, mostly from the initial assessment visit $(448,376,99.6 \%)$. Our analyses were limited to the 417,580 individuals of European ancestry with 25OHD concentrations available, of whom 318,851 are unrelated (gcta --rel-cutoff 0.05).

Genome-wide association study (GWAS) analysis. Figure 1 provides a graphical summary of the GWAS and post-GWAS analyses detailed below. To identify genetic variants associated with $25 \mathrm{OHD}$ levels, we performed a linear mixed model GWAS implemented in fastGWA ${ }^{62}$. fastGWA is a tool implemented in GCTA ${ }^{63}$ for mixed linear model (MLM)-based GWAS. It uses a sparse genomic relationship matrix (GRM) to account for genetic structure within the cohort, making it a resource-efficient method for the analysis of large data sets like the UK Biobank ${ }^{62}$. The sparse GRM was generated for UKB individuals of European ancestry using HapMap3 SNPs.

We applied a rank-based inverse-normal transformation (RINT) to the phenotype (vitamin D 25OHD levels) and fit age at time of assessment, sex, assessment month, assessment centre, supplement-intake information, genotyping batch and the first 40 ancestry PCs as covariates in the model (see Supplementary Methods for more details).

To identify independent associations, we a conducted a conditional and joint (COJO; gcta --cojo-slct) analysis ${ }^{18}$ of the GWAS results, accounting for the correlation structure between SNPs within a $10-\mathrm{Mb}$ window (COJO default parameter) and using a random subset of 20,000 unrelated Europeans from the UKB as linkage disequilibrium (LD) reference. For comparison, we used PLINK1.9 (--clump) ${ }^{64}$ to identify regional lead SNPs for genome-wide significant index variants (--clump-p1 5e-8) and variants were clumped with this lead SNP if they were located less than $10 \mathrm{Mb}$ (--clump-kb 10000) away from, and with $r^{2}>0.01$ (--clump-r2 0.01 ) with the index variant. To identify previously unreported associations, we a conducted a COJO analysis that conditioned (gcta --cojo-cond) on the six loci previously reported as genome-wide significant (rs2282679, rs10741657, rs12785878, rs10745742, rs8018720, rs6013897) $8,20,65$.

Meta-analysis. The largest GWAS for 25OHD to date, from the SUNLIGHT consortium $^{8}$, used BMI as a covariate, hence we also generated UKB results including BMI in the model and used those for meta-analysis. In addition, the UKB GWAS results used for meta-analysis differed from the reported GWAS results in that 25OHD levels were natural-log transformed, and supplement intake was not included as a covariate. Before meta-analysis, we imputed the SUNLIGHT summary statistics $\left(2,579,297\right.$ SNPs) with ImpG ${ }^{66}$. After data management, we used a sample size-based approach ${ }^{67}$ to perform the meta-analysis (Supplementary Methods) on 6,912,294 SNPs that were shared between the data sets.

Relationship between vitamin D and body mass index traits. High BMI is associated with lower concentrations of $25 \mathrm{OHD}^{10}$. For this reason, previous GWAS of 25OHD have included BMI as covariate in their analyses ${ }^{8}$. However, given that BMI is a highly heritable trait, covariate adjustment can induce collider bias ${ }^{15}$ and affect downstream analyses. To better understand the relationship between $25 \mathrm{OHD}$ and BMI, we estimated the phenotypic and genetic correlation between them and used generalised summary-data-based Mendelian randomisation (GSMR) ${ }^{16}$ to test for statistical evidence for putative causal effects between the two traits. We confirmed through simulation that the MR regression statistic is not biased by sample overlap (Supplementary Note 5). SNP instruments were selected with the default settings of the built-in GSMR clumping step (which is less stringent than used in our clumping protocol because GSMR accounts for residual correlation between SNP instruments). In addition, we conducted a multi-trait conditional and joint (mtCOJO) analysis ${ }^{16}$ to condition the 25OHD GWAS results on BMI GWAS summary statistics generated with the $\mathrm{UKB}^{17}$, an approach that was shown in simulations to be robust to induced collider bias when conditioning on a correlated trait $^{16}$. A random subset of 20,000 unrelated individuals of European ancestry from the UKB was used as LD reference in the mtCOJO analysis.

Heritability and SNP-based heritability. Our UKB sample included a set of 58,738 individuals who were related with coefficient of relationship $(r)>0.2$ to at least one other person in the set (all relatives). Among these, there was a set including all pairs with $0.4<r<0.6$ (1st degree), and a set including all pairs with $0.2<r<0.3$ (2nd degree). We used these sets to estimate heritability of RINT (25OHD) levels (gcta --reml). To estimate SNP-based heritability, we drew a random subset $(N \sim 50,000)$, selected so that no pair of individuals had $r>0.05$. We used a model that fits a single random genetic effect with a single genomic relationship matrix (GRM) constructed from all SNPs ${ }^{68}$, and also a GREML-LDMS model ${ }^{69}$ that fits ten random genetic effects and hence ten GRM (gcta --reml --mgrm). The ten GRM were constructed from SNPs annotated to five MAF (0.01-0.1, 0.1-0.2, 0.2-0.3, 0.3-0.4 and 0.4-0.5) bins each divided into two by median LD score of the SNPs within the bin. The LD score of a SNP is a measure of the common genetic variation tagged by a SNP. The sum of the estimates for each MAF-LD bin is an estimate of the total SNP-based heritability. Under a neutral model, each of the five MAF bins is expected to explain $20 \%$ of the variance. Analyses were conducted with and without BMI as a covariate, and genetic correlation between 25OHD and BMI was estimated in a bivariate GREML analysis (gcta --reml-bivar). In addition, we estimated the genetic correlation and the genetic variance explained by $25 \mathrm{OHD}$ levels assessed in summer and winter (see definitions in vQTL and seasonal analysis section below), using bivariate GREML. Heritability and SNP-based heritability estimated as part of the GWAS analysis using fastGWA are also reported. Finally, we estimated SNP-based heritability by LD score regression ${ }^{28}$ (software default settings for European ancestry samples), SBayesR ${ }^{13}$ and SBayesS ${ }^{13}$ from GWAS summary statistics. From SBayesS, we also estimate the polygenicity $(\pi)$ and selection (S) parameters.

Replication and out-of-sample genetic risk prediction. We used the QIMR Brisbane-based twin and family sample $(N=1632 \text { unrelated })^{6}$ for replication analyses. Samples were collected between May 1992 and January 2014, mostly from South-East Queensland (latitude $27^{\circ} \mathrm{S}$ ). At this latitude, there is sufficient UVR to allow for vitamin D synthesis throughout the year ${ }^{70}$. Legal guardians gave written, informed consent prior to inclusion and testing. Studies were approved by the Human Research Ethics Committee of the QIMR Berghofer Medical Research Institute. Additional details of this study are provided elsewhere (25OHD assay 
methods ${ }^{6}$ and genotyping on HumanCoreExome-12v1-0_C or IlluminaHuman610W-Quad bead chip and quality $\left.\operatorname{control}^{71}\right)$. Genotypes were imputed to phase 3 version 5 of the 1000 Genomes Build37 (hg19) 21 . The phenotype analysed was RINT(25OHD) pre-regressed on sex, age, month of collection, ten ancestry PCs and imputation batch. Association analysis was conducted in PLINK for the genome-wide significant COJO SNPs from the UKB analysis. We tested for the same sign of effect size between the UKB and the QIMR results. Next, using the sample, we conducted polygenic score analyses. Using only SNPs present in the Brisbane cohort, we selected independently associated SNPs from the UKB cohort in order to conduct standard $P$-value thresholding PRS analysis, choosing a range of $P$-value thresholds $\left(P<5 \times \times 10^{-8}, P<1 \times 10^{-5}, P<0.001, P<0.01, P<\right.$ $0.05, P<0.1, P<0.5, P<1)$ and calculating polygenic scores for each individual in the QIMR cohort. We also calculated polygenic scores from SNP weights estimated by $\mathrm{COJO}^{72}$, SBayesR ${ }^{22}$ and SBayesS ${ }^{13}$. The Bayesian methods better account for the complex relationship between strength of association of, and correlation between, SNP effect sizes. For each set of polygenic scores, we estimated the proportion of variance explained by the scores in linear regression. We repeated these analyses in a sample of 1632 individuals selected from the UKB, whose PC1 value was just outside our cut-off for white European (referred to as the UKBR sample; Supplementary Methods).

Functional mapping and annotation of GWAS. We conducted a number of analyses to annotate the 25OHD GWAS results. First, we used the FUMA online pipeline $^{23}$ to obtain gene-based, gene-set and tissue-specific annotations. Second, we used functional annotations provided in the LDSC software to partition SNPbased heritability into 53 functional categories ${ }^{25}$. Annotations included elements, such as UCSC, UTRs, promoter and intronic regions, conserved regions and functional genomic annotations constructed using ENCODE ${ }^{73}$ and Roadmap Epigenomics Consortium data ${ }^{74}$. Third, we assessed the SNP-based heritability enrichment associated with different cell types. Specifically, we applied LDSC analysis to the GWAS summary statistics using scores associated with cell-typespecific expression (as provided in the LDSC software) ${ }^{75}$.

To help prioritise putative causal genes with expression underlying 25OHD levels, we used the summary-data-based Mendelian randomisation method (SMR) ${ }^{26}$. SMR integrates GWAS and eQTL (expression quantitative trait loci, SNPs associated with gene expression) results with the aim of identifying pleiotropic or causal associations between a trait of interest and gene expression. We used eQTLs derived by the eQTLGen consortium from gene expression in whole blood ${ }^{27}$, using the a large sample for blood eQTLs $(N=31,684)$, identifying 15,504 genome-wide significant eQTLs. In general, SNPs controlling variation in one tissue are found to control variation in other tissues ${ }^{76}$, hence using a large eQTL dataset is the most powerful approach. Moreover, blood is a relevant tissue for vitamin D-related gene transcription ${ }^{77}$. Other relevant tissues are the liver, skin and, given our hypotheses about the relationship between $25 \mathrm{OHD}$ and psychiatric disorders, the brain. To capture tissue-specific eQTLs in these tissues, we used GTEX eQTL data sets, despite the fact that these data sets are much smaller than the eQTLGen sample (sunexposed skin, $N=369$; non-sun-exposed skin, $N=335$; liver, $N=153$; 16 brain regions, $N$ between 80 and 154). In addition, we used eQTLs identified in pre-frontal cortex $(N=1866)$ from the PsychENCODE project ${ }^{78}$, and foetal brain samples $(N=$ 120) from O'Brien et al. ${ }^{79}$. SMR significant results were declared at $P_{\mathrm{SMR}}<0.05 / \mathrm{M}$ per tissue, where $\mathrm{M}$ is the number SMR tests performed (i.e., the number of gene probes tested for the tissue, Supplementary Data 9). While significant SMR test results implicate a role for the eQTL gene, SNPs passing the conservative SMR heterogeneity in dependent instruments (HEIDI) test $\left(P_{\text {HEIDI }}>0.05\right)$ have robust support for the direct causal or pleiotropic relationships of the trait-associated SNPs influencing gene expression.

\section{Genetic correlations and putative causal relationships with other traits} Published epidemiological studies have provided an extensive set of hypotheses about causal relationships between vitamin $\mathrm{D}$ and a range of phenotypes ${ }^{80}$, including psychiatric and brain-related disorders ${ }^{81}$ including intelligence ${ }^{31}$. To characterise the relationship between vitamin $\mathrm{D}$ and psychiatric traits, we conducted two sets of analyses. First, we used bivariate LD score regression ${ }^{28}$ to estimate the genetic correlation between vitamin $\mathrm{D}$ and psychiatric traits using the GWAS summary statistics generated with the UKB dataset and GWAS summary statistics that are available for attention deficit/hyperactivity disorder (ADHD) ${ }^{82}$, Alzheimer's disease $(\mathrm{AD})^{83}$, major depression $(\mathrm{MD})^{84}$, schizophrenia $(\mathrm{SCZ})^{85}$, bipolar disorder (BIP $)^{86}$ and autism spectrum disorder (ASD ${ }^{87}$. In addition, we obtained genetic correlation estimates between vitamin $\mathrm{D}$ and 746 traits available through the LD Hub database ${ }^{30}$. Second, we conducted generalised summary Mendelian randomisation (GSMR) analyses ${ }^{16}$ to assess if there was any statistical evidence that observed correlations could be explained by a causal relationship for seventeen traits (Fig. 5). GSMR analyses were conducted as described above (see "Relationship between vitamin D and body mass index traits" section), with significance declared at $0.05 / 18=0.003$. For any significant associations observed with GSMR, we confirmed our conclusions using the two-sample MR (2SMR) method $^{29}$, which implements a range of MR models that can adjust for the potential influence of pleiotropy (MR Egger, weighted mean, inverse variance weighted, simple mode and weighted mode).
Proxy-environment vQTL and seasonal analysis. 25OHD concentration is known to be affected by season of measurement, but other environmental factors may also impact $25 \mathrm{OHD}$ measures. We conducted a genome-wide vQTL analysis $^{12}$, an approach to detect presence of genotype-by-environment interaction in the absence of measurement or knowledge of the interacting environmental risk factor, to identify SNPs associated with variance in 25OHD. Specifically, we used the Levene's median test implemented in OSCA ${ }^{88}$. Following the guidelines of Wang et al. ${ }^{12}$, we (1) adjusted 25OHD levels for selected covariates (see below), (2) removed outliers more than $5 \mathrm{SD}$ from the mean and (3) standardised the residuals to have mean 0 and variance 1 . Each step was performed within one of eight groups defined based on sex (male vs. female) and supplement intake (none, other, vitamin $\mathrm{D}$ or missing). This approach removed both the mean effect of covariates and the mean and variance differences between gender and supplement- intake groups, while retaining other distributional properties of the measure. Covariates included in the phenotype pre-regression were age at assessment, assessment month, assessment centre, genotyping batch and the first 40 PCs. To avoid spurious associations due to coincidence of low-frequency variants with phenotype out$\operatorname{liers}^{12}$, this analysis was restricted to SNPs with MAF $>0.05$. To identify nearindependent vQTLs, we clumped the vQTL GWAS results with PLINK1.9 (--clump) as above, using a $5 \mathrm{Mb}$ window as recommended ${ }^{12}$.

To assess if significant vQTL associations reflected a GxE with season of testing we conducted season-stratified GWAS and compared the results with the VQTL GWAS results. Specifically, we stratified the UKB cohort into two groups after visual inspection of the mean 25OHD concentrations per month (Supplementary Fig. 1b). We defined two discrete time periods in order to retain the maximum sample size, but optimise comparisons between months with higher and lower mean 25OHD concentrations: (a) Winter-individuals assessed Dec-Apr ( $=$ 162,591), and (b) Summer-individuals assessed Jun-Oct $(\mathrm{N}=177,082)$. Individuals with vitamin D levels assessed in the months of May and November were not included in these analyses. The two season-stratified GWAS (winter and summer) were conducted as the main GWAS (i.e., linear mixed model implemented in fastGWA, with the same covariates included in the model). In addition, we conducted a GxE analysis using PLINK1.959 (--gxe) to include an interaction term with season of blood draw. For this analysis, the phenotype (25OHD levels) was processed as described for the VQTL analysis.

Reporting summary. Further information on research design is available in the Nature Research Reporting Summary linked to this article.

\section{Data availability}

Genome-wide association summary statistics generated with the three levels of BMI correction (i.e., with and without BMI as covariate, and conditioned on BMI) are available for download from https://cnsgenomics.com/content/data. Results for the UKB GWAS of BMI used for conditional analysis are also available from the same website. UK Biobank data were obtained through direct application to the UK Biobank. The SUNLIGHT data were downloaded from https://drive.google.com/drive/folders/ 0BzYDtCo_doHJRFRKRoltZHZWZjQ. Functional annotations to partition SNP-based heritability with LDSC were downloaded from https://data.broadinstitute.org/ alkesgroup/LDSCORE/. eQTL data were downloaded from http://www.eqtlgen.org/ciseqtls.html and https://cnsgenomics.com/software/smr/\#DataResource. GWAS summary statistics used for bidirectional GSMR were downloaded from https://walters.psycm.cf.ac. uk (schizophrenia), https://cnsgenomics.com/content/data (type II diabetes), https://ctg. $\mathrm{cncr.nl/software/summary \_ statistics} \mathrm{(Alzheimer's} \mathrm{disease;} \mathrm{fluid} \mathrm{intelligence;} \mathrm{ADHD),}$ https://www.thessgac.org/data (educational attainment), https://www.med.unc.edu/pgc/ download-results/ (bipolar disorder; autism spectrum disorder), http://plaza.umin.ac.jp/ yokada/datasource/software.htm (rheumatoid arthritis), ftp://ftp.ebi.ac.uk/pub/ databases/gwas/summary_statistics/vanderHarstP_29212778_GCST005194 (coronary artery disease), https://www.ibdgenetics.org/downloads.html (inflammatory bowel disease). All other data are contained in the article and its supplementary information, or are available on request.

Received: 27 November 2019; Accepted: 3 March 2020; Published online: 02 April 2020

\section{References}

1. Holick, M. F. Vitamin D deficiency. N. Engl. J. Med. 357, 266-281 (2007).

2. Institute of Medicine. Dietary Reference Intakes for Calcium and Vitamin D (National Academies Press, 2010)

3. Lips, P. Worldwide status of vitamin D nutrition. J. Steroid Biochem. Mol. Biol. 121, 297-300 (2010).

4. Karohl, C. et al. Heritability and seasonal variability of vitamin D concentrations in male twins. Am. J. Clin. Nutr. 92, 1393-1398 (2010).

5. Mills, N. T. et al. Heritability of transforming growth factor-beta 1 and tumor necrosis factor-receptor type 1 expression and vitamin D levels in healthy adolescent twins. Twin Res. Hum. Genet. 18, 28-35 (2015). 
6. Mitchell, B. L. et al. Half the genetic variance in vitamin D concentration is shared with skin colour and sun exposure genes. Behav. Genet. 49, 386-398 (2019).

7. Jiang, X., Kiel, D. P. \& Kraft, P. The genetics of vitamin D. Bone 126, 59-77 (2018).

8. Jiang, X. et al. Genome-wide association study in 79,366 European-ancestry individuals informs the genetic architecture of 25-hydroxyvitamin D levels. Nat. Commun. 9, 260 (2018).

9. Sudlow, C. et al. UK biobank: an open access resource for identifying the causes of a wide range of complex diseases of middle and old age. PLoS Med. 12, e1001779 (2015).

10. Hypponen, E. \& Power, C. Hypovitaminosis D in British adults at age $45 \mathrm{y}$ : nationwide cohort study of dietary and lifestyle predictors. Am. J. Clin. Nutr. 85, 860-868 (2007)

11. Eyles, D. W. et al. The association between neonatal vitamin D status and risk of schizophrenia. Sci. Rep. 8, 17692 (2018).

12. Wang, H. et al. Genotype-by-environment interactions inferred from genetic effects on phenotypic variability in the UK Biobank. Sci. Adv. 5, eaaw3538 (2019).

13. Zeng, J. et al. Bayesian analysis of GWAS summary data reveals differential signatures of natural selection across human complex traits and functional genomic categories. Preprint at https://www.biorxiv.org/content/10.1101/ 752527v1 (2019).

14. International HapMapConsortium et al. Integrating common and rare genetic variation in diverse human populations. Nature 467, 52-58 (2010).

15. Day, F. R., Loh, P. R., Scott, R. A., Ong, K. K. \& Perry, J. R. A robust example of collider bias in a genetic association study. Am. J. Hum. Genet. 98, 392-393 (2016).

16. Zhu, Z. et al. Causal associations between risk factors and common diseases inferred from GWAS summary data. Nat. Commun. 9, 224 (2018).

17. Xue, A. et al. Genome-wide association analyses identify 143 risk variants and putative regulatory mechanisms for type 2 diabetes. Nat. Commun. 9, 2941 (2018).

18. Yang, J. et al. Conditional and joint multiple-SNP analysis of GWAS summary statistics identifies additional variants influencing complex traits. Nat. Genet. 44, 369-75, S1-3 (2012).

19. Manousaki, D. et al. Low-frequency synonymous coding variation in CYP2R1 has large effects on vitamin D levels and risk of multiple sclerosis. Am. J. Hum. Genet. 101, 227-238 (2017).

20. Wang, T. J. et al. Common genetic determinants of vitamin D insufficiency: a genome-wide association study. Lancet 376, 180-188 (2010).

21. Genomes Project, C. et al. A global reference for human genetic variation. Nature 526, 68-74 (2015).

22. Lloyd-Jones, L. R. et al. Improved polygenic prediction by Bayesian multiple regression on summary statistics. Nat. Commun. 10, 5086 (2019).

23. Watanabe, K., Taskesen, E., van Bochoven, A. \& Posthuma, D. Functional mapping and annotation of genetic associations with FUMA. Nat. Commun. 8, 1826 (2017).

24. GTEx Consortium. Genetic effects on gene expression across human tissues. Nature 550, 204 (2017).

25. Finucane, H. K. et al. Partitioning heritability by functional annotation using genome-wide association summary statistics. Nat. Genet. 47, 1228-1235 (2015).

26. Zhu, Z. et al. Integration of summary data from GWAS and eQTL studies predicts complex trait gene targets. Nat. Genet. 48, 481-487 (2016).

27. Võsa, U. et al. Unraveling the polygenic architecture of complex traits using blood eQTL metaanalysis. Preprint at https://www.biorxiv.org/content/ $10.1101 / 447367 \mathrm{v} 1$ (2018).

28. Bulik-Sullivan, B. et al. An atlas of genetic correlations across human diseases and traits. Nat. Genet. 47, 1236-1241 (2015).

29. Hemani, G. et al. The MR-Base platform supports systematic causal inference across the human phenome. eLife 7, e34408 (2018).

30. Zheng, J. et al. LD Hub: a centralized database and web interface to perform LD score regression that maximizes the potential of summary level GWAS data for SNP heritability and genetic correlation analysis. Bioinformatics 33, 272-279 (2017).

31. Sniekers, S. et al. Genome-wide association meta-analysis of 78,308 individuals identifies new loci and genes influencing human intelligence. Nat. Genet. 49, 1107-1112 (2017).

32. Tomalik-Scharte, D. et al. Impaired hepatic drug and steroid metabolism in congenital adrenal hyperplasia due to P450 oxidoreductase deficiency. Eur. J. Endocrinol. 163, 919-924 (2010).

33. Caffeine Genetics Consortium. et al. Genome-wide meta-analysis identifies six novel loci associated with habitual coffee consumption. Mol. Psychiatry 20, 647-656 (2015).

34. Canela-Xandri, O., Rawlik, K. \& Tenesa, A. An atlas of genetic associations in UK Biobank. Nat. Genet. 50, 1593-1599 (2018).
35. Kuchenbaecker, K. et al. The transferability of lipid loci across African, Asian and European cohorts. Nat. Commun. 10, 4330 (2019).

36. Mechin, M. C. et al. The peptidylarginine deiminases expressed in human epidermis differ in their substrate specificities and subcellular locations. Cell Mol. Life Sci. 62, 1984-1995 (2005).

37. Marenholz, I. et al. Meta-analysis identifies seven susceptibility loci involved in the atopic march. Nat. Commun. 6, 8804 (2015).

38. Prassas, I., Eissa, A., Poda, G. \& Diamandis, E. P. Unleashing the therapeutic potential of human kallikrein-related serine proteases. Nat. Rev. Drug Discov. 14, 183-202 (2015).

39. Thyssen, J. P., Bikle, D. D. \& Elias, P. M. Evidence that loss-of-function filaggrin gene mutations evolved in Northern Europeans to favor intracutaneous vitamin D3 production. Evol. Biol. 41, 388-396 (2014).

40. Thyssen, J. P. et al. Skin barrier abnormality caused by filaggrin (FLG) mutations is associated with increased serum 25-hydroxyvitamin D concentrations. J. Allergy Clin. Immunol. 130, 1204-1207 e2 (2012).

41. Eckhart, L. et al. Histidase expression in human epidermal keratinocytes: regulation by differentiation status and all-trans retinoic acid. J. Dermatol. Sci. 50, 209-215 (2008)

42. Welsh, M. M. et al. A role for ultraviolet radiation immunosuppression in non-melanoma skin cancer as evidenced by gene-environment interactions. Carcinogenesis 29, 1950-1954 (2008).

43. Landeck, L. et al. The effect of epidermal levels of urocanic acid on 25hydroxyvitamin D synthesis and inflammatory mediators upon narrowband UVB irradiation. Photodermatol. Photoimmunol. Photomed. 32, 214-223 (2016).

44. de Fine Olivarius, F. et al. Urocanic acid isomers: relation to body site, pigmentation, stratum corneum thickness and photosensitivity. Arch. Dermatol. Res. 289, 501-505 (1997).

45. Wang, Z. et al. Human UGT1A4 and UGT1A3 conjugate 25-hydroxyvitamin D3: metabolite structure, kinetics, inducibility, and interindividual variability. Endocrinology 155, 2052-2063 (2014).

46. Wong, T. et al. Polymorphic human sulfotransferase $2 \mathrm{~A} 1$ mediates the formation of 25-hydroxyvitamin D3-3-O-sulfate, a major circulating vitamin D metabolite in humans. Drug Metab. Dispos. 46, 367-379 (2018).

47. Kurogi, K., Sakakibara, Y., Suiko, M. \& Liu, M. C. Sulfation of vitamin D3related compounds-identification and characterization of the responsible human cytosolic sulfotransferases. FEBS Lett. 591, 2417-2425 (2017).

48. Nozawa, T. et al. Genetic polymorphisms of human organic anion transporters OATP-C (SLC21A6) and OATP-B (SLC21A9): allele frequencies in the Japanese population and functional analysis. J. Pharm. Exp. Ther. 302, 804-813 (2002).

49. Mueller, J. W., Gilligan, L. C., Idkowiak, J., Arlt, W. \& Foster, P. A. The regulation of steroid action by sulfation and desulfation. Endocr. Rev. 36, 526-563 (2015)

50. Hypponen, E. \& Boucher, B. J. Adiposity, vitamin D requirements, and clinical implications for obesity-related metabolic abnormalities. Nutr. Rev. 76 678-692 (2018).

51. Lee, M. J. et al. Vitamin D deficiency in northern Taiwan: a community-based cohort study. BMC Public Health 19, 337 (2019).

52. Eyles, D. W., Burne, T. H. J. \& McGrath, J. J. Vitamin D, effects on brain development, adult brain function and the links between low levels of vitamin D and neuropsychiatric disease. Front. Neuroendocrinol. 34, 47-64 (2013).

53. Adamson, J. et al. Correlates of vitamin D in psychotic disorders: a comprehensive systematic review. Psychiatry Res. 249, 78-85 (2017).

54. McGrath, J. J. et al. Neonatal vitamin D status and risk of schizophrenia: a population-based case-control study. Arch. Gen. Psychiatry 67, 889-894 (2010).

55. Fry, A. et al. Comparison of sociodemographic and health-related characteristics of UK Biobank participants with those of the general population. Am. J. Epidemiol. 186, 1026-1034 (2017).

56. McCarthy, S. et al. A reference panel of 64,976 haplotypes for genotype imputation. Nat. Genet. 48, 1279-1283 (2016).

57. Consortium, U. K. et al. The UK10K project identifies rare variants in health and disease. Nature 526, 82-90 (2015).

58. Bycroft, C. et al. The UK Biobank resource with deep phenotyping and genomic data. Nature 562, 203-209 (2018).

59. Chang, C. C. et al. Second-generation PLINK: rising to the challenge of larger and richer datasets. Gigascience 4, 7 (2015).

60. Genomes Project, C. et al. A map of human genome variation from population-scale sequencing. Nature 467, 1061-1073 (2010).

61. Fry, D., Almond, R., Moffat, S., Gordon, M., \& Singh, P. B. UK Biobank Biomarker Project: Companion Document to Accompany Serum Biomarker Data. UK Biobank Document Showcase (2019). Available at: https://biobank. ndph.ox.ac.uk/showcase/showcase/docs/serum_biochemistry.pdf. Accessed Mar 2020.

62. Jiang, L. et al. A resource-efficient tool for mixed model association analysis of large-scale data. Nat. Genet. 51, 1749-1755 (2019). 
63. Yang, J., Lee, S. H., Goddard, M. E. \& Visscher, P. M. Genome-wide complex trait analysis (GCTA): methods, data analyses, and interpretations. Methods Mol. Biol. 1019, 215-236 (2013).

64. Purcell, S. et al. PLINK: a tool set for whole-genome association and population-based linkage analyses. Am. J. Hum. Genet. 81, 559-575 (2007).

65. Ahn, J. et al. Genome-wide association study of circulating vitamin D levels. Hum. Mol. Genet. 19, 2739-2745 (2010).

66. Pasaniuc, B. et al. Fast and accurate imputation of summary statistics enhances evidence of functional enrichment. Bioinformatics 30, 2906-2914 (2014).

67. Willer, C. J., Li, Y. \& Abecasis, G. R. METAL: fast and efficient meta-analysis of genomewide association scans. Bioinformatics 26, 2190-2191 (2010).

68. Lee, S. H., Yang, J., Goddard, M. E., Visscher, P. M. \& Wray, N. R. Estimation of pleiotropy between complex diseases using single-nucleotide polymorphism-derived genomic relationships and restricted maximum likelihood. Bioinformatics 28, 2540-2542 (2012).

69. Yang, J. et al. Genetic variance estimation with imputed variants finds negligible missing heritability for human height and body mass index. Nat. Genet. 47, 1114-1120 (2015).

70. Kimlin, M. G. et al. The contributions of solar ultraviolet radiation exposure and other determinants to serum 25-hydroxyvitamin D concentrations in Australian adults: the AusD Study. Am. J. Epidemiol. 179, 864-874 (2014).

71. Medland, S. E. et al. Common variants in the trichohyalin gene are associated with straight hair in Europeans. Am. J. Hum. Genet. 85, 750-755 (2009).

72. Yang, J., Lee, S. H., Goddard, M. E. \& Visscher, P. M. GCTA: a tool for genome-wide complex trait analysis. Am. J. Hum. Genet. 88, 76-82 (2011).

73. Consortium, E. P. An integrated encyclopedia of DNA elements in the human genome. Nature 489, 57-74 (2012).

74. Bernstein, B. E. et al. The NIH roadmap epigenomics mapping consortium. Nat. Biotechnol. 28, 1045-1048 (2010).

75. Finucane, H. K. et al. Heritability enrichment of specifically expressed genes identifies disease-relevant tissues and cell types. Nat. Genet. 50, 621-629 (2018).

76. Qi, T. et al. Identifying gene targets for brain-related traits using transcriptomic and methylomic data from blood. Nat. Commun. 9, 2282 (2018).

77. Neme, A. et al. In vivo transcriptome changes of human white blood cells in response to vitamin D. J. Steroid Biochem. Mol. Biol. 188, 71-76 (2019).

78. Wang, D. et al. Comprehensive functional genomic resource and integrative model for the human brain. Science 362, eaat8464 (2018).

79. O'Brien, H. E. et al. Expression quantitative trait loci in the developing human brain and their enrichment in neuropsychiatric disorders. Genome Biol. 19, 194 (2018).

80. Theodoratou, E., Tzoulaki, I., Zgaga, L. \& Ioannidis, J. P. Vitamin D and multiple health outcomes: umbrella review of systematic reviews and metaanalyses of observational studies and randomised trials. BMJ 348, g2035 (2014).

81. Groves, N. J., McGrath, J. J. \& Burne, T. H. Vitamin D as a neurosteroid affecting the developing and adult brain. Annu. Rev. Nutr. 34, 117-141 (2014).

82. Demontis, D. et al. Discovery of the first genome-wide significant risk loci for attention deficit/hyperactivity disorder. Nat. Genet. 51, 63-75 (2019).

83. Marioni, R. E. et al. GWAS on family history of Alzheimer's disease. Transl. Psychiatry 8, 99 (2018).

84. Howard, D. M. et al. Genome-wide meta-analysis of depression identifies 102 independent variants and highlights the importance of the prefrontal brain regions. Nat. Neurosci. 22, 343-352 (2019).

85. Pardinas, A. F. et al. Common schizophrenia alleles are enriched in mutationintolerant genes and in regions under strong background selection. Nat. Genet. 50, 381-389 (2018).

86. Stahl, E. A. et al. Genome-wide association study identifies 30 loci associated with bipolar disorder. Nat. Genet. 51, 793-803 (2019).

87. Grove, J. et al. Identification of common genetic risk variants for autism spectrum disorder. Nat. Genet. 51, 431-444 (2019).

88. Zhang, F. et al. OSCA: a tool for omic-data-based complex trait analysis. Genome Biol. 20, 107 (2019).

\section{Acknowledgements}

This study was carried out under the generic approval from the NHS National Research Ethics Service and conducted using the UK Biobank resource under projects 12505 and 10214. We thank the UKB participants, project team and funders for providing this important research resource. We thank the eQTLGen consortium for providing the cis eQTL dataset based on $N=32 \mathrm{~K}$ participants. We thank 23andMe for the use of GWAS summary statistics for major depression that include data from 23andMe. We would like to thank the research participants and employees of 23andMe for making this work possible. The Genotype-Tissue Expression (GTEx) Project was supported by the Common Fund of the Office of the Director of the National Institutes of Health, and by NCI, NHGRI, NHLBI, NIDA, NIMH and NINDS. Funding for the QIMR sample was provided by the Australian National Health and Medical Research Council (NHMRC) and further supported by NHMRC Project Grants $(1007677,1099709)$ and a John Cade Fellowship (1056929). NHMRC also support Naomi Wray $(1113400,1078901)$, Peter Visscher $(1113400,1078037)$ and Jian Yang (1113400). Jian Yang is supported by the Australian Research Council (FT180100186). John McGrath is supported by the Danish National Research Foundation (Niels Bohr Professorship, the NHMRC (John Cade Fellowship 1056929). John McGrath, Darryl Eyles and Thomas Burne are employed by The Queensland Centre for Mental Health Research which receives core funding from the Queensland Health. Darryl Eyles is supported by the NHMRC $(1124724,1124721,1141699)$. Brittany Mitchell received financial support from the Queensland University of Technology.

\section{Author contributions}

J.A.R., J.J.M. and N.R.W. conceived the study and designed the analyses. J.A.R., T.L., Z.Q and B.M. conducted the analyses. K.E.K. and J.S. performed the initial preparation and quality control of the UK Biobank data. A.X., Y.H., Z.Z., J.Z., H.W., A.A.E.V. and G.Z. provided support in analysis implementation. J.F., D.E. and T.H.J.B. helped with interpretation of identified loci. N.G.M. provided the QIMR cohort, and B.M. and G.Z. conducted the analyses based on this sample. P.M.V. and J.Y. provided advice on analyses and interpretation of the results. J.A.R., J.J.M. and N.R.W. wrote the paper with the participation of all the authors. All authors reviewed and approved the final paper.

\section{Competing interests}

The authors declare no competing interests.

\section{Additional information}

Supplementary information is available for this paper at https://doi.org/10.1038/s41467020-15421-7.

Correspondence and requests for materials should be addressed to N.R.W. or J.J.M.

Peer review information Nature Communications thanks Dominic Furniss, Fernando Rivadeneira and Xia Jiang for their contribution to the peer review of this work.

Reprints and permission information is available at http://www.nature.com/reprints

Publisher's note Springer Nature remains neutral with regard to jurisdictional claims in published maps and institutional affiliations.

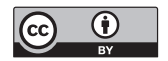

Open Access This article is licensed under a Creative Commons Attribution 4.0 International License, which permits use, sharing, adaptation, distribution and reproduction in any medium or format, as long as you give appropriate credit to the original author(s) and the source, provide a link to the Creative Commons license, and indicate if changes were made. The images or other third party material in this article are included in the article's Creative Commons license, unless indicated otherwise in a credit line to the material. If material is not included in the article's Creative Commons license and your intended use is not permitted by statutory regulation or exceeds the permitted use, you will need to obtain permission directly from the copyright holder. To view a copy of this license, visit http://creativecommons.org/ licenses/by/4.0/.

(C) The Author(s) 2020 